\title{
A rare pathogen Raoultella planticola caused urinary tract infection in child with congenital anomalies of kidney and urinary tract: case report
}

\author{
Yuya Fukuda $^{1} \wedge$, Shuhei Adachi ${ }^{1}$, Makoto Saito $^{1}$, Reikichi Shoji $^{1}$, Atsuo Togashi ${ }^{2}$ \\ ${ }^{1}$ Department of Pediatrics, Steel Memorial Muroran Hospital, Muroran, Japan; ${ }^{2}$ Department of Pediatrics, Iwamizawa Municipal General Hospital, \\ Iwamizawa, Japan \\ Correspondence to: Yuya Fukuda. Department of Pediatrics, Steel Memorial Muroran Hospital, 45-1 Chiribetsu, Muroran, Hokkaido, 050-0076, Japan. \\ Email: fuku.da1f9@gmail.com.
}

\begin{abstract}
Raoultella planticola was previously considered an environmental organism in soil, water, and plants. However, several cases of human infection have recently been reported in association with $R$. planticola, some of which have been life-threatening. Most cases were in adults with reduced immunity, with few cases in children. To our knowledge, there have only been two reported cases of urinary tract infection (UTI) caused by $R$. planticola in children, including one case of cystitis. Here, we present the first case of UTI caused by $R$. planticola with congenital anomalies of kidney and urinary tract (CAKUT) in a 4-month-old male infant. The patient presented to the emergency department with fever and was diagnosed with UTI. We started third-generation cephalosporins empirically for gram-negative bacteria in the urine, presuming infection with Escherichia coli. On day 1, the patient's fever resolved immediately. On day 2, urine culture was positive for a rare pathogen, $R$. planticola, and we narrowed antibiotics to first-generation cephalosporins. The patient's fever did not return and he was discharged on day 7 . The patient was seen in the clinic 1 week after discharge, with complete resolution of symptoms. Magnetic resonance urography and dynamic renal scintigraphy performed 2 months after discharge revealed severe bilateral hydronephroureter and obstruction of urine flow in the right kidney. As of 6 months after UTI onset, we have continued low-dose cephalexin $(10 \mathrm{mg} / \mathrm{kg})$ to prevent the recurrence of UTI and there has been no recurrence. As in this case, children with UTI caused by $R$. planticola may be associated with CAKUT; therefore, we should actively screen to detect CAKUT. Patients with CAKUT are at high risk of UTI recurrence, so long-term use of unnecessary broad-spectrum antibiotics should be avoided to prevent antimicrobial resistance. However, R. planticola infection is sometimes life-threatening. Hence, it is also important to use sufficiently strong antibiotics for an appropriate period. Although the optimal management of $R$. planticola infection in children has not been clearly established, we suggest that we can treat UTI caused by $R$. planticola mainly using first-generation cephalosporins.
\end{abstract}

Keywords: Raoultella planticola; urinary tract infection; child; hydronephrosis; case report

Submitted Apr 17, 2021. Accepted for publication Jul 22, 2021.

doi: $10.21037 / \mathrm{tp}-21-170$

View this article at: https://dx.doi.org/10.21037/tp-21-170

\footnotetext{
^ ORCID: 0000-0001-9413-7224.
} 


\section{Introduction}

Raoultella planticola is a gram-negative, oxidase-negative, aerobic bacterium that was previously considered an environmental organism present in soil, water, and plants. However, several cases of human infection in association with $R$. planticola have recently been reported, including cases of bacteremia, pancreatitis, endocarditis, cholangitis, and urinary tract infection (UTI), mostly in adult patients. Here, we describe the case of a 4-month-old male infant with UTI and congenital anomalies of kidney and urinary tract (CAKUT) caused by $R$. planticola. Children with UTI caused by $R$. planticola are extremely rare, and this was the first case of UTI with CAKUT. As in this case, children with UTI caused by $R$. planticola may be associated with CAKUT, which should be actively screened.

We present the following case in accordance with the CARE reporting checklist (available at https://dx.doi. org/10.21037/tp-21-170).

\section{Case presentation}

A 4-month-old male infant presented to the emergency department of Steel Memorial Muroran Hospital (Japan) with a 6-hour history of fever, shaking chills, and lethargy. The patient was born in another hospital and was previously healthy, with an uneventful medical history or family history. Vital signs showed a temperature of $38.0^{\circ} \mathrm{C}$, pulse of 150 beats per minute, and respiratory rate of 50 breaths per minute. Physical examination was unremarkable except for a bad mood. Blood tests showed a white blood cell (WBC) count 17,920 cells/ $\mu \mathrm{L}$ (absolute neutrophil count, 11,200 cells $/ \mu \mathrm{L}$ ), C-reactive protein (CRP) $1.40 \mathrm{mg} / \mathrm{dL}$, and procalcitonin $0.49 \mathrm{ng} / \mathrm{mL}$. Urinalysis showed WBC more than 100 per high-power field (HPF), 1 to 4 red blood cells/HPF, and $3+$ bacteria using a scale of 0 to $4+$. Abdominal ultrasound revealed severe right hydronephrosis [Society for Fetal Urology (SFU) grade 3-4], mild left hydronephrosis (SFU grade 1), and bilateral hydroureter (Figure 1). Gram staining of urine specimens revealed clumps of gram-negative rods (Figure 2). We diagnosed febrile UTI and started intravenous cefotaxime, presuming an infection with Escherichia coli. On day 1, the patient's fever resolved immediately. On day 2, urine culture obtained by catheterization on admission was positive for greater than $1 \times 10^{7}$ colony forming units per $\mathrm{mL}$ of $R$. planticola, which was susceptible to nearly all antibiotics except ampicillin and piperacillin (Table 1). We changed antibiotics from cefotaxime to cefazolin and the patient's fever did not return. Bacterial species identification and susceptibility testing were performed using an automated identification system (VITEK $^{\circledR} 2$, bioMérieux). On day 4, follow-up laboratory tests revealed an improved WBC count of 8,470 cells $/ \mu \mathrm{L}$, CRP $0.74 \mathrm{mg} / \mathrm{dL}$, and urinalysis with fewer than $5 \mathrm{WBCs} /$ HPF. On day 7, the patient was discharged and started on cephalexin. The patient was seen in the clinic 1 week after discharge with complete resolution of symptoms. Two months after discharge, we performed magnetic resonance urography (MRU), dynamic renal scintigraphy, and voiding cystourethrography (VCUG). MRU revealed bilateral hydronephroureter, with marked dilation of the upper right ureter, but there was no ectopic ureter (Figure 3). Dynamic renal scintigraphy showed obstruction of urine flow in the right kidney, and normal flow in the left kidney. No vesicoureteral reflux was detected on VCUG. As of 6 months after UTI onset, we have continued low-dose cephalexin $(10 \mathrm{mg} / \mathrm{kg})$ to prevent the recurrence of UTI and there has been no recurrence. We will continue to follow up once every few months and consider surgical treatment if UTI recurs.

All procedures performed in studies involving human participants were in accordance with the ethical standards of the institutional and/or national research committee(s) and with the Helsinki Declaration (as revised in 2013). Written informed consent was obtained from the child's parent.

\section{Discussion}

UTI is a common but serious bacterial infection in childhood, and E. coli is the most common pathogen in the pediatric population, accounting for approximately $80-90 \%$ of total cases $(1,2)$. Non-E. coli pathogens including Enterococcus species, Klebsiella species, Proteus species, and Enterobacter species, are often detected in patients with CAKUT (3-5). To the best of our knowledge, this is the first report of UTI with CAKUT in children, caused by a rare pathogen, $R$. planticola. Tests in this patient revealed severe bilateral hydronephroureter and right ureteral obstruction, which could provide the underlying conditions for growth of $R$. planticola in the urine. We should actively perform testing for patients with UTI caused by $R$. planticola whether they have CAKUT. This patient was born in another hospital; therefore, we could not determine whether the patient had prenatal hydronephrosis. It was unclear whether this $R$. planticola infection could have been prevented by administering prophylactic antibiotics. 

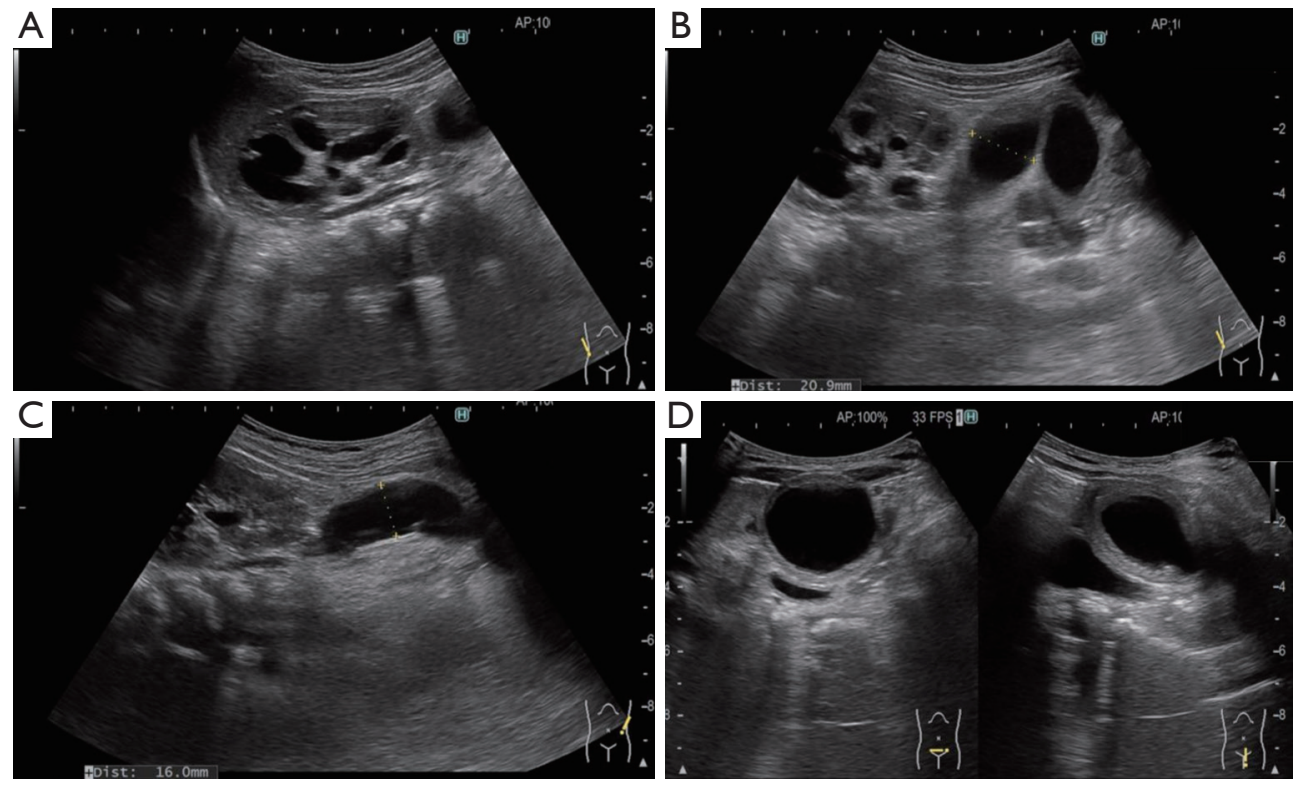

Figure 1 Abdominal ultrasound. (A) right hydronephrosis [Society for Fetal Urology (SFU) grade 3-4]; (B) right upper urinary tract dilation; (C) left hydronephrosis (SFU grade 1) and upper urinary tract dilation; (D) bladder and right lower urinary tract dilation).

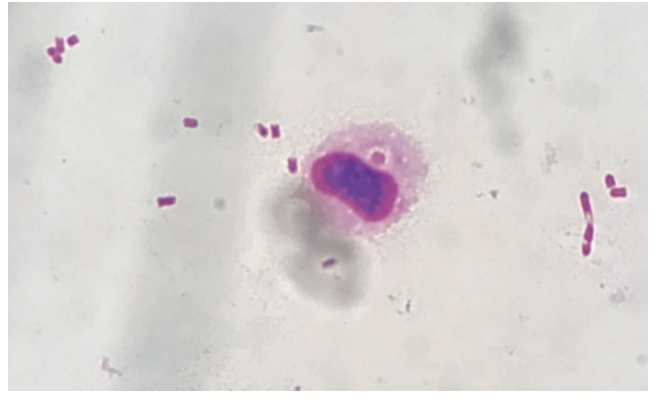

Figure 2 Gram staining of urine specimens showing phagocytosed gram-negative $\operatorname{rod}(\times 1,000)$.

Until the late 1990s, Raoultella species were classified as Klebsiella species. In 2001, comparative analysis of $16 \mathrm{~S}$ ribosomal RNA and rpoB gene sequences established Raoultella as a new genus in the family Enterobacteriaceae (6). According to the current taxonomy, the genus Raoultella contains four species: $R$. planticola, $R$. ornithinolytica, $R$. terrigena and $R$. electrica. $R$. planticola is the most frequently described species and most frequently associated with human infections in the genus (7). The first case of human infection with $R$. planticola was reported in 1984 in a patient with sepsis admitted to an intensive care unit in France (8). $R$. planticola has gradually become recognized as an important pathogen associated with sometimes life-threatening infections in adults.

Past reports of infections associated with $R$. planticola among pediatric patients have been limited to cases of endocarditis, bacteremia, oral mucositis, conjunctivitis, cystitis, neutropenic fever, central venous line exit-site infection, and peritonitis $(9,10)$. Many of these patients had severe complications, including Burkitt's lymphoma, B cell lymphoblastic lymphoma, complicated meconium ileus, and Meckel's diverticulitis/perforation. No deaths have been reported in children with $R$. planticola infection; however, those patients may have weakened immunity or severe underlying diseases. Therefore, it is important to not only use sufficiently strong antibiotics for an appropriate period but also to avoid the unnecessary use of broad-spectrum antibiotics to prevent development of antibiotic-resistant bacteria. $R$. planticola is usually susceptible to nearly all antibiotics except aminopenicillins, as in the current case (9). However, it should be noted that multidrug-resistant strains of $R$. planticola have been reported (11-14).

To our knowledge, there have been two cases of UTI caused by $R$. planticola in children. Howell et al. reported a 2-month-old febrile female infant who was treated with ceftriaxone during admission and was started on cephalexin upon discharge for 10 days (10). The other case was cystitis in a 16-month-old male child with rhabdomyosarcoma of the bladder neck. The patient received cefotaxime and 
Table 1 Susceptibility testing for $R$. planticola of current case

\begin{tabular}{|c|c|c|}
\hline Antibiotics & MIC $(\mu \mathrm{g} / \mathrm{mL})$ & Susceptibility \\
\hline ABPC & $\geq 32$ & $\mathrm{R}$ \\
\hline PIPC & 16 & $\mathrm{R}$ \\
\hline CEZ & $\leq 4$ & S \\
\hline CTM & $\leq 8$ & S \\
\hline CTX & $\leq 1$ & $S$ \\
\hline CFPM & $\leq 1$ & S \\
\hline CAZ & $\leq 1$ & S \\
\hline $\mathrm{CMZ}$ & $\leq 1$ & S \\
\hline FMOX & $\leq 2$ & S \\
\hline IPM & 0.5 & S \\
\hline MEPM & $\leq 0.25$ & S \\
\hline AZT & $\leq 1$ & $S$ \\
\hline SBT/ABPC & 4 & S \\
\hline GM & $\leq 1$ & S \\
\hline AMK & $\leq 2$ & S \\
\hline MINO & 2 & $S$ \\
\hline $\mathrm{CP}$ & $\leq 2$ & S \\
\hline CPFX & $\leq 0.25$ & S \\
\hline LVFX & $\leq 0.12$ & $S$ \\
\hline ST & $\leq 20$ & S \\
\hline
\end{tabular}

MIC, minimum inhibitory concentration; R, resistant; S, suscept ible; ABPC, ampicillin; PIPC, piperacillin; CEZ, cefazolin; CTM, cefotiam; CFPM, cefepime; CAZ, ceftazidime; CMZ, cefmetazole; FMOX, flomoxef; IPM, imipenem; MEPM, meropenem; AZT, aztreonam; SBT/ABPC, sulbactam/ampicillin; GM, gentamicin; AMK, amikacin; MINO, minocycline; CP, chloramphenicol; CPEX, ciprofloxacin; LVFX, levofloxacin; ST, sulfamethoxazole-trimethoprim.

ampicillin/sulbactam for 6 days, with subsequent oral cefpodoxime for 4 days (15). Different from these cases, we immediately narrowed the third-generation cephalosporins to first-generation cephalosporins on day 2, with subsequent treatment success. Considering the severe bilateral hydronephroureter and right ureteral obstruction, this patient is at high risk of UTI recurrence. Hence, avoiding long-term use of broad-spectrum antibiotics is beneficial in this case. Although the appropriate management of $R$. planticola infections in children has not been clearly established, we suggest that $R$. planticola infection can

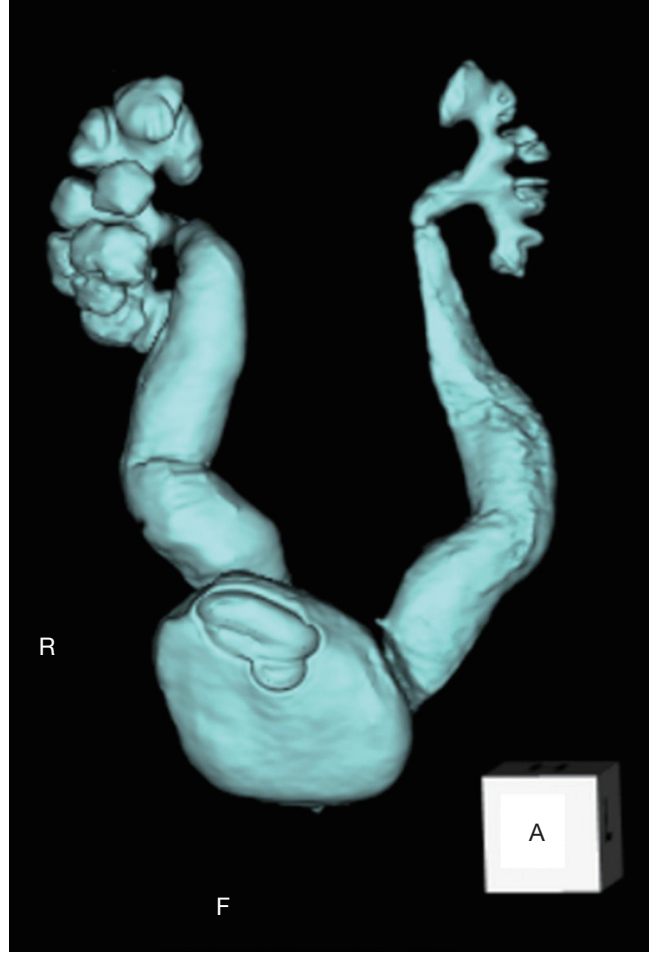

Figure 3 Bilateral hydronephrosis and hydroureter in magnetic resonance urography.

be mainly treated with first-generation cephalosporins. However, this was only one case; additional cases must be evaluated to determine whether treatment for $R$. planticola infection using first-generation cephalosporins is effective.

\section{Acknowledgments}

We would like to thank Kazuyuki Nishinaka for performing MRU, dynamic renal scintigraphy, and VCUG. We thank Analisa Avila, MPH, ELS, from Edanz (https://jp.edanz. com/ac) for editing a draft of this manuscript.

Funding: None.

\section{Footnote}

Reporting Checklist: The authors have completed the CARE reporting checklist. Available at https://dx.doi. org/10.21037/tp-21-170

Conflicts of Interest: All authors have completed the ICMJE uniform disclosure form (available at https://dx.doi. org/10.21037/tp-21-170). The authors have no conflicts of 
interest to declare.

Ethical Statement: The authors are accountable for all aspects of the work in ensuring that questions related to the accuracy or integrity of any part of the work are appropriately investigated and resolved. All procedures performed in studies involving human participants were in accordance with the ethical standards of the institutional and/or national research committee(s) and with the Helsinki Declaration (as revised in 2013). Written informed consent was obtained from the child's parent.

Open Access Statement: This is an Open Access article distributed in accordance with the Creative Commons Attribution-NonCommercial-NoDerivs 4.0 International License (CC BY-NC-ND 4.0), which permits the noncommercial replication and distribution of the article with the strict proviso that no changes or edits are made and the original work is properly cited (including links to both the formal publication through the relevant DOI and the license). See: https://creativecommons.org/licenses/by-nc-nd/4.0/.

\section{References}

1. Korbel L, Howell M, Spencer JD. The clinical diagnosis and management of urinary tract infections in children and adolescents. Paediatr Int Child Health 2017;37:273-9.

2. Morello W, La Scola C, Alberici I, et al. Acute pyelonephritis in children. Pediatr Nephrol 2016;31:1253-65.

3. Stein R, Dogan HS, Hoebeke P, et al. Urinary tract infections in children: EAU/ESPU guidelines. Eur Urol 2015;67:546-58.

4. Megged O. Staphylococcus aureus urinary tract infections in children are associated with urinary tract abnormalities and vesico-ureteral reflux. Pediatr Nephrol 2014;29:269-72.

5. Bell LE, Mattoo TK. Update on childhood urinary

Cite this article as: Fukuda Y, Adachi S, Saito M, Shoji R, Togashi A. A rare pathogen Raoultella planticola caused urinary tract infection in child with congenital anomalies of kidney and urinary tract: case report. Transl Pediatr 2021;10(9):2387-2391. doi: $10.21037 /$ tp-21-170 tract infection and vesicoureteral reflux. Semin Nephrol 2009;29:349-59.

6. Drancourt M, Bollet C, Carta A, et al. Phylogenetic analyses of Klebsiella species delineate Klebsiella and Raoultella gen. nov., with description of Raoultella ornithinolytica comb. nov., Raoultella terrigena comb. nov. and Raoultella planticola comb. nov. Int J Syst Evol Microbiol 2001;51:925-32.

7. Sękowska A. Raoultella spp.-clinical significance, infections and susceptibility to antibiotics. Folia Microbiol (Praha) 2017;62:221-7.

8. Freney J, Fleurette J, Gruer LD, et al. Klebsiella trevisanii colonisation and septicaemia. Lancet 1984;1:909.

9. Pacilli M, Nataraja RM. Raoultella planticola associated with Meckel's diverticulum perforation and peritonitis in a child: Case report and systematic review of the paediatric literature. J Infect Public Health 2019;12:605-7.

10. Howell C, Fakhoury J. A case of Raoultella planticola causing a urinary tract infection in a pediatric patient. Transl Pediatr 2017;6:102-3.

11. Chen X, Guo S, Liu D, et al. Neonatal septicemia caused by a rare pathogen: Raoultella planticola - a report of four cases. BMC Infect Dis 2020;20:676.

12. Xu M, Xie W, Fu Y, et al. Nosocomial pneumonia caused by carbapenem-resistant Raoultella planticola: a case report and literature review. Infection 2015;43:245-8.

13. Chen DQ, Song JL, Tang HX, et al. Extensively drugresistant Raoultella planticola carrying multiple resistance genes including blaNDM-1. JMM Case Rep 2014;1

14. Castanheira M, Deshpande LM, DiPersio JR, et al. First descriptions of blaKPC in Raoultella spp. (R. planticola and R. ornithinolytica): report from the SENTRY Antimicrobial Surveillance Program. J Clin Microbiol 2009;47:4129-30.

15. Yoon JH, Ahn YH, Chun JI, et al. Acute Raoultella planticola cystitis in a child with rhabdomyosarcoma of the bladder neck. Pediatr Int 2015;57:985-7. 